\title{
Photometry of V 1794 Cygni between 1975 and 1995^
}

\author{
L. Jetsu ${ }^{1,8}$, I. Tuominen ${ }^{2}$, B.W. Bopp ${ }^{3}$, O.V. Ezhkova ${ }^{4}$, K.N. Grankin ${ }^{4}$, T. Hackman ${ }^{1}$, P.A. Heckert ${ }^{5}$, \\ M.A. Ibragimov ${ }^{4}$, V.B. Kondratiev ${ }^{4}$, S.Yu. Mel'nikov ${ }^{4}$, T. Oja ${ }^{6}$, H.L. Nations ${ }^{7}$, and V.S. Shevchenko ${ }^{4}$ \\ 1 Observatory, P.O. Box 14, University of Helsinki, FIN-00014, Finland \\ 2 Astronomy Division, University of Oulu, P.O. Box 3000, FIN-90410 Oulu, Finland \\ 3 Ritter Observatory, University of Toledo, Toledo, Ohio 43606, U.S.A. \\ 4 Astronomical Institute of the Academy of Sciences of Uzbekistan, Astronomicheskaya 33, Tashkent 700052, Uzbekistan \\ 5 Department of Chemistry and Physics, Western Carolina University, Cullowhee, NC 28723, U.S.A. \\ 6 Astronomical Observatory, Box 515, S-751 20 Uppsala, Sweden \\ 7 Physics Department, College of Charleston, Charleston, South Carolina 29424, U.S.A. \\ 8 NORDITA, Blegdamsvej 17, DK-2100 Copenhagen, Denmark
}

Received June 8, 1998; accepted August 6, 1999

\begin{abstract}
Six years of new photometry of V 1794 Cyg is combined with the previously published data. These data are processed into two decades of homogeneous standard Johnson $U B V R I$ photometry, which contains 1207, 1837, 1927, 867 and 75 observations in $U, B, V, R$ and $I$, respectively.
\end{abstract}

Key words: stars: individual: V 1794 Cyg (HD 199178); variables - techniques: photometric

\section{Introduction}

The FK Comae-type stars were defined as single and rapidly rotating $\mathrm{G}-\mathrm{K}$ giants with strong chromospheric and transition region UV emission (Bopp \& Rucinski 1981; Bopp \& Stencel 1981). The rapidly rotating post-mainsequence star V 1794 Cyg (HD 199178, G5III-IV, $P_{\text {phot }}=$ 3 d $\left.337484, v \sin i=65.4 \mathrm{~km} \mathrm{~s}^{-1}\right)$ is one of the original candidates for this class (Herbig 1958; Jetsu et al. 1990a, Paper I; Fekel 1997). V 1794 Cyg seems to have no binary companion, because the radial velocity is nearly constant (Huenemoerder 1986: $v_{\text {rad }}=-27.9 \pm 1.6 \mathrm{~km} \mathrm{~s}^{-1}$ ). Jetsu et al. (1990b, Paper II) combined new data with all earlier photometry of V 1794 Cyg (Bopp 1982; Bopp et al. 1983; Kaluzny 1984; Nations \& Seeds 1986; Huovelin et al. 1987). Those data between 1975 and 1989 were

Send offprint requests to: jetsu@gstar.astro.helsinki.fi

* Table 4 is only available in electronic form at the CDS via anonymous ftp to cdsarc.u-strasbg.fr (130.79.128.5) or via http://cdsweb.u-strasbg.fr/Abstract.html, http://www.edpsciences.org analysed in Paper I. Since then additional photometry of V 1794 Cyg have been published (e.g. Heckert \& Stewart 1992; Rodono \& Cutispoto 1992; Dempsey et al. 1992). We present new photometry of V 1794 Cyg made between 1989 and 1995 at the Mount Hopkins Observatory, the Mount Maidanak Observatory, the Mount Laguna Observatory, the Kvistaberg Observatory, and the Royal Swedish La Palma Observatory. This paper presents the methods applied to guarantee the homogeneity of these data between 1975 and 1995, which are analysed in Jetsu et al. (1999, Paper III). The following topics are discussed: the division of the V 1794 Cyg data into 114 subsets (SET: Sect. 2), the secondary comparison stars $\left(C_{2}\right.$ : Sect. 3$)$, the primary comparison stars $\left(C_{1}\right.$ : Sect. 4$)$, and the previous and the new photometry of V 1794 Cyg (Sects. 5 and 6 ). The photometric measurements of the primary and secondary comparison stars have been compiled into Tables 1 and 2, respectively. The necessary SET information of the V 1794 Cyg data is summarized in Table 3. Finally, the collected photometry of V 1794 Cyg is given Table 4, which is only available in electronic form (see Grewing et al. 1992).

\section{Observations}

The typical subset length of $30^{\mathrm{d}}$ ensures an adequate light curve phase coverage for V $1794 \mathrm{Cyg}$. These light curves do not seem to change significantly during this time interval (Paper II). The first observing time of each subset determines the unique SET sequence for any part of collected photometry. Our Table 3 summarizes the relevant information of all 114 subsets: observing time interval, observatory, $C_{1}, C_{2}$, reference and number of observing nights. The collected photometry of V $1794 \mathrm{Cyg}$ is 
Table 1. The $U B V R I$ magnitudes of all $C_{2}$, and their weighted means

\begin{tabular}{|c|c|c|c|c|c|}
\hline $\begin{array}{c}16 \text { Cyg B } \\
U \\
\end{array}$ & $B$ & $V$ & $R$ & $I$ & Reference \\
\hline $7.060 \pm 0.026$ & $6.860 \pm 0.019$ & $6.200 \pm 0.017$ & & & Johnson \& Morgan 1953 \\
\hline $7.090 \pm 0.031$ & $6.900 \pm 0.023$ & $6.210 \pm 0.022$ & & & Tolbert 1964 \\
\hline $7.070 \pm 0.019$ & $6.880 \pm 0.014$ & $6.220 \pm 0.011$ & & & Argue 1966 \\
\hline \multirow{10}{*}{$7.072 \pm 0.043$} & $6.855 \pm 0.043$ & $6.191 \pm 0.013$ & $5.750 \pm 0.016$ & $5.408 \pm 0.016$ & Johnson et al. 1966 \\
\hline & $6.898 \pm 0.022$ & $6.238 \pm 0.020$ & $5.692 \pm 0.023$ & $5.341 \pm 0.027$ & Moffett \& Barnes 1979 \\
\hline & & $6.217 \pm 0.010$ & & & Tedesco et al. 1982 \\
\hline & & $6.230 \pm 0.009$ & & & Olsen 1983 \\
\hline & & $6.200 \pm 0.010$ & & & Campins et al. 1985 \\
\hline & $6.910 \pm 0.028$ & $6.250 \pm 0.020$ & $5.716 \pm 0.028$ & $5.350 \pm 0.028$ & Rakos \& Franz 1988 \\
\hline & & $6.232 \pm 0.004$ & & & Fabregat \& Reglero 1990 \\
\hline & & $6.242 \pm 0.008$ & & & Stetson 1991 \\
\hline & $6.876 \pm 0.017$ & $6.215 \pm 0.016$ & & & Turon et al. 1992 \\
\hline & & $6.238 \pm 0.004$ & & & Skiff 1993 \\
\hline $7.071 \pm 0.010$ & $6.882 \pm 0.015$ & $6.230 \pm 0.013$ & $5.728 \pm 0.025$ & $5.383 \pm 0.031$ & Weighted mean for 16 Cyg B \\
\hline \multicolumn{6}{|l|}{57 Cyg } \\
\hline & $4.630 \pm 0.023$ & $4.780 \pm 0.018$ & & & Ljunggren \& Oja 1964 \\
\hline $4.044 \pm 0.023$ & $4.625 \pm 0.023$ & $4.766 \pm 0.022$ & $4.833 \pm 0.023$ & $4.968 \pm 0.025$ & Johnson et al. 1966 \\
\hline $4.025 \pm 0.023$ & $4.635 \pm 0.017$ & $4.760 \pm 0.014$ & & & Eggen 1968 \\
\hline \multirow[t]{3}{*}{$4.100 \pm 0.026$} & $4.660 \pm 0.021$ & $4.800 \pm 0.019$ & & & Crawford et al. 1971 \\
\hline & & $4.740 \pm 0.010$ & & & Straizys et al. 1989 \\
\hline & $4.640 \pm 0.009$ & $4.774 \pm 0.007$ & & & Turon et al. 1992 \\
\hline $4.053 \pm 0.031$ & $4.639 \pm 0.009$ & $4.766 \pm 0.017$ & $4.833 \pm 0.023$ & $4.968 \pm 0.025$ & Weighted mean for $57 \mathrm{Cyg}$ \\
\hline \multicolumn{6}{|l|}{ SAO 50257} \\
\hline & & $6.660 \pm 0.010$ & & & Straizys et al. 1989 \\
\hline & $7.554 \pm 0.152$ & $6.634 \pm 0.022$ & & & Turon et al. 1992 \\
\hline & $7.554 \pm 0.152$ & $6.656 \pm 0.010$ & & & Weighted mean for SAO 50257 \\
\hline \multicolumn{6}{|l|}{ SAO 50326} \\
\hline $9.990 \pm 0.010$ & $8.730 \pm 0.008$ & $7.540 \pm 0.006$ & & & McClure 1970 \\
\hline \multirow[t]{2}{*}{$10.010 \pm 0.027$} & $8.720 \pm 0.020$ & $7.560 \pm 0.008$ & & & Landolt 1975 \\
\hline & & $7.540 \pm 0.010$ & & & Straizys et al. 1989 \\
\hline $9.992 \pm 0.007$ & $8.729 \pm 0.003$ & $7.546 \pm 0.009$ & & & Weighted mean for SAO 50326 \\
\hline
\end{tabular}

given in Table 4. It contains the $U B V R I$ magnitudes of V 1794 Cyg (Cols. 3-7), their subset numbers and heliocentric julian dates (Cols. 1-2: SET and HJD), and the detected flares (Col. 8: F, see also Paper III: Sect. 3.1).

\section{Secondary comparison stars $\left(C_{2}\right)$}

The $C_{2}$ of differential photometry is observed to confirm the constant brightness of $C_{1}$, and to determine the brightness of $C_{1}$. Even for a $C_{2}$ of unknown brightness, the differential magnitudes $\Delta m_{C_{1}-C_{2}}$ can confirm the shortterm constant brightness of $C_{1}$. But this does not test the long-term constant brightness of $C_{1}$. For example, a low inclination late-type spotted $C_{1}$ with long-term variability might not exhibit short-term variability. If the brightness of $C_{2}$ is known, the $C_{1}$ brightness is obtained, and the long-term constancy of $C_{1}$ can eventually be verified with respect to the other available data. It is crucial for the long-term differential photometry of any variable star (O) that the constant brightness of the chosen $C_{1}$ and $C_{2}$ combination is confirmed with high accuracy. Such a combination should be consistently used in all subsequent differential photometry of $\mathrm{O}$. To achieve this goal, we compiled numerous references of the $U B V R I$ magnitudes (and errors) for all $C_{1}$ and $C_{2}$ of $\mathrm{V} 1794 \mathrm{Cyg}$ (Tables 1 and 2). Furthermore, Table 2 contains new data for the two most frequently used $C_{1}$ (SAO 50313 and SAO 50205). We will show that the best combination for V $1794 \mathrm{Cyg}$ would be $C_{2}=57$ Cyg with $C_{1}=\mathrm{SAO} 50313$ or SAO 50205 .

Earlier $C_{1}$ and $C_{2}$ combinations for V 1794 Cyg appear arbitrary (see Table 3). The position of V 1794 Cyg in the vicinity of the North America Nebula (NGC 7000) and the Pelican Nebula (IC 5070) offers numerous $C_{1}$ and $C_{2}$ alternatives. Four secondary (16 Cyg B, $57 \mathrm{Cyg}$, SAO 50257, SAO 50326) and three primary comparison stars (SAO 50313, SAO 50205, SAO 50260) have been used. Furthermore, the primary comparison star SAO 50313 was once used as $C_{2}(\mathrm{SET}=38)$. The $C_{2}$ of each SET is specified in Table 3 (Col. 5 ), where "Absolute" denotes absolute photometry. No $C_{2}$ was observed or specified during some subsets.

\subsection{Secondary comparison star 16 Cyg B}

16 Cyg B (HD 186427, SAO 31899) was used as a $C_{2}$ only once $(\mathrm{SET}=28)$. The long-term $U B V$ are constant (Table 1), but the $R I$ show some scatter. The Cousins 

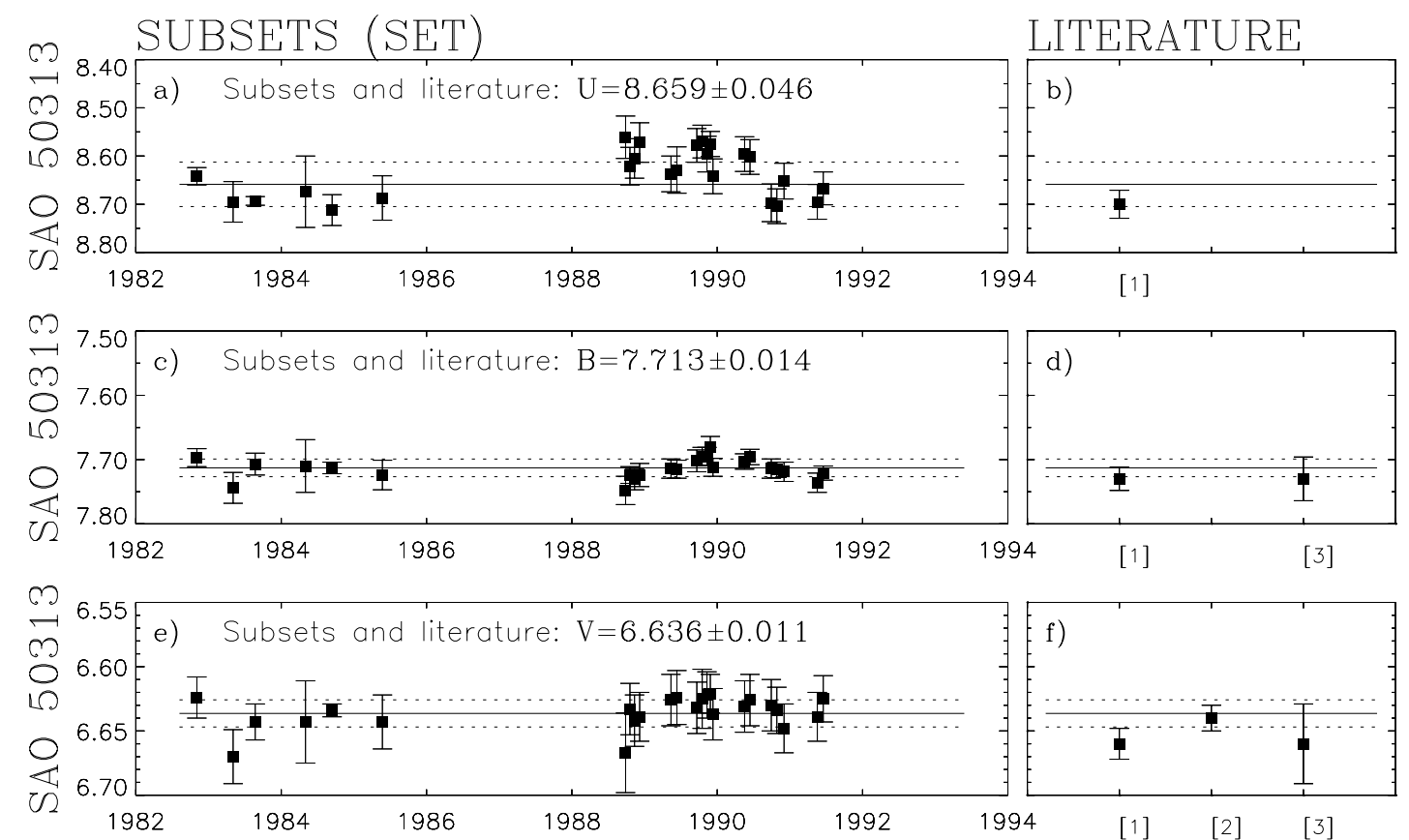

[1] $=$ Landolt 1975 [2]=Straizys et al. 1989 [3]=Turon et al. 1992

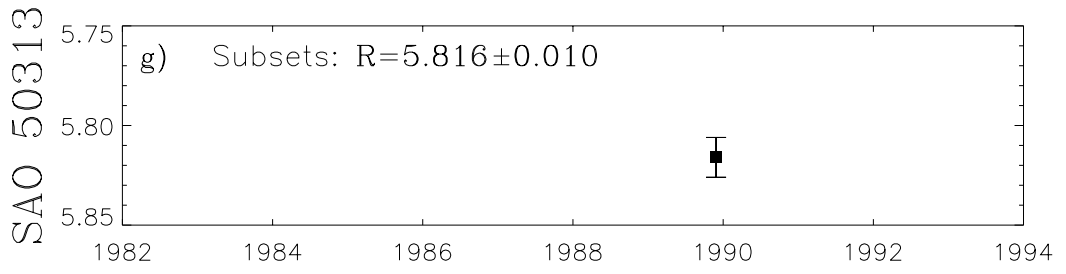

Fig. 1. aceg) The $U B V R$ magnitudes of SAO 50313 during individual subsets (Table 2: $\pm 1 \sigma$ errors). The weighted mean and error are outlined with the continuous and the dashed line, respectively. bdf) Other measurements with respect to the reference number (i.e. no epoch)

$R I$ by Rakos \& Franz (1988) transformed to the standard Johnson system (Bessell 1979) agree with Moffett \& Barnes (1979). Thus the RI by Johnson et al. (1966) induce the large errors of the long-term mean $R I$ (Table 1$)$. 16 Cyg B is a close "solar analog"-candidate (Neckel 1986: G3v, $\left.T_{\text {eff }}=5735 \mathrm{~K}, B-V=0.66, U-B=0.20\right)$. Since the upper limit of the solar luminosity variations is \pm 0 . 001 (Willson \& Hudson 1991), detection of photometric variability in this "solar analog"-candidate would be surprising. 16 Cyg B has been the standard star in many photometric studies (e.g. Moffett \& Barnes 1979; Skiff 1993). The angular separation of $\Delta_{\text {sep }}=13.6$ between 16 Cyg B and V 1794 Cyg requires a large extinction correction, and thus it is an inconvenient $C_{2}$ for differential photometry.

\subsection{Secondary comparison star 57 Cyg}

57 Cyg (HD 199081, SAO 50180, $\Delta_{\text {sep }}=0.2$ ) has been the most frequently used $C_{2}$ for $\mathrm{V} 1794 \mathrm{Cyg}$. The $U B V$ mea- surements indicate constant long-term brightness. But the $R I$ have been measured only once (Johnson et al. 1966). 57 Cyg is an early-type B5V spectroscopic binary with $P_{\text {orb }}=2.854825$, but not an eclipsing binary (Hilditch 1973; Batten et al. 1978; Giuricin et al. 1984). Since the masses are nearly equal $\left(M_{1} / M_{2}=1.125\right)$, and the orbital inclination is $i \simeq 48^{\circ}$, the upper limit for $B$ changes is $\sim 0$. 02 (Hilditch 1973). The differential $U B V$ magnitudes $\Delta m_{C_{1}-C_{2}}$ of our APT photometry revealed no periodicity with $P_{\text {orb }}$, nor any significant periodicity between 0.4 and $50^{\mathrm{d}}$. Finally, we confirmed the $P_{\text {orb }}$ for the $v_{\text {rad }}$ data in Hilditch (1973). That 57 Cyg was successfully used as $C_{2}$ by Percy \& Welch (1983) in studying the low amplitude photometric variability in early-type supergiants also supports short-term constant brightness. Our APT differential $U B V$ photometry of SAO 50313 and SAO 50205 with respect to this $C_{2}$ confirmed constant short-term brightness. Because the $U B V$ magnitudes are accurate and the $R I$ magnitudes are available, we conclude that $57 \mathrm{Cyg}$ is currently the best $C_{2}$ choice for V $1794 \mathrm{Cyg}$. Furthermore, 

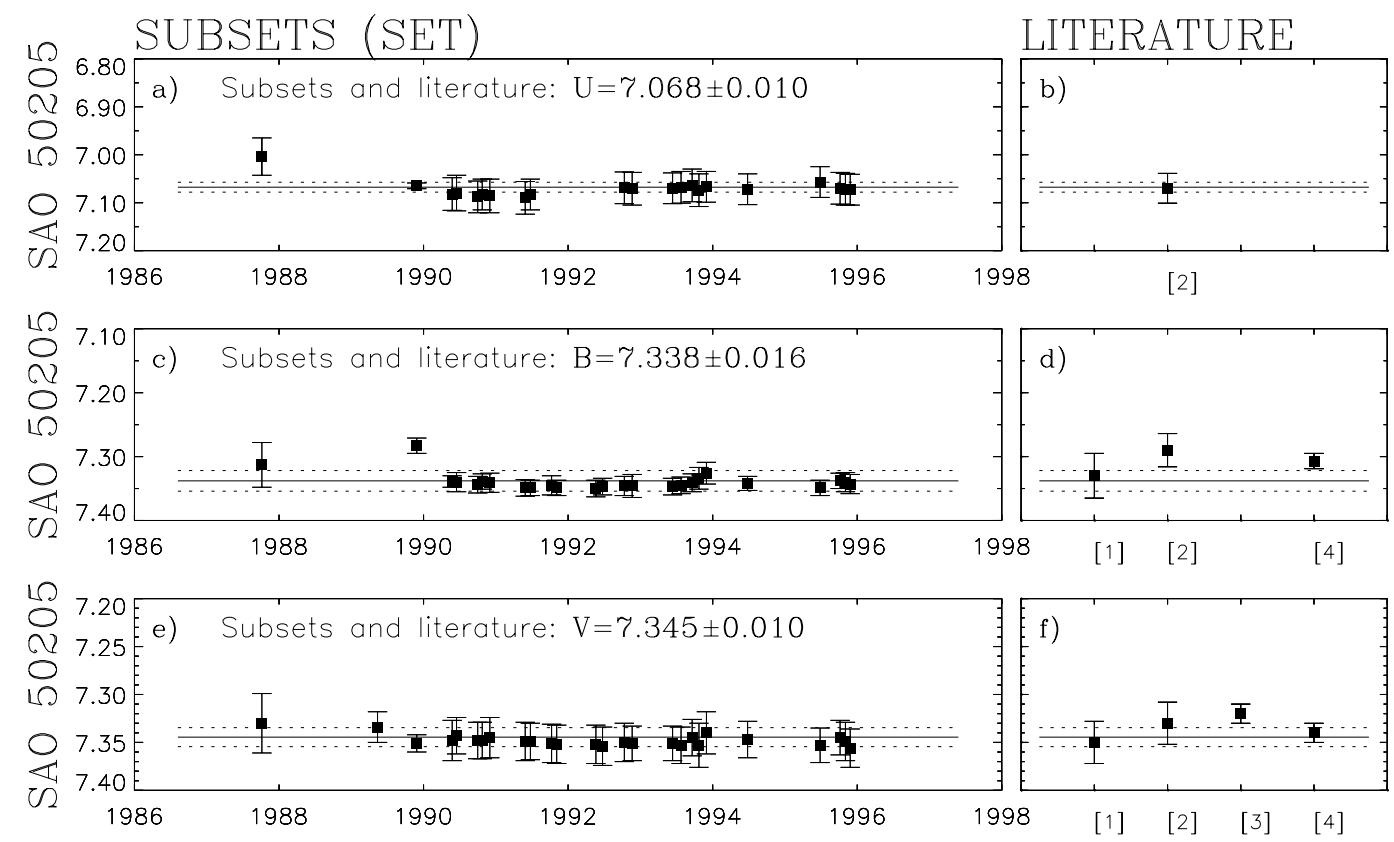

$[1]=$ junggren \& Oja 1964 [2]=Deutschman et al. 1976 [3]=Straizys et al. 1989 [4]=Turon et al. 1992
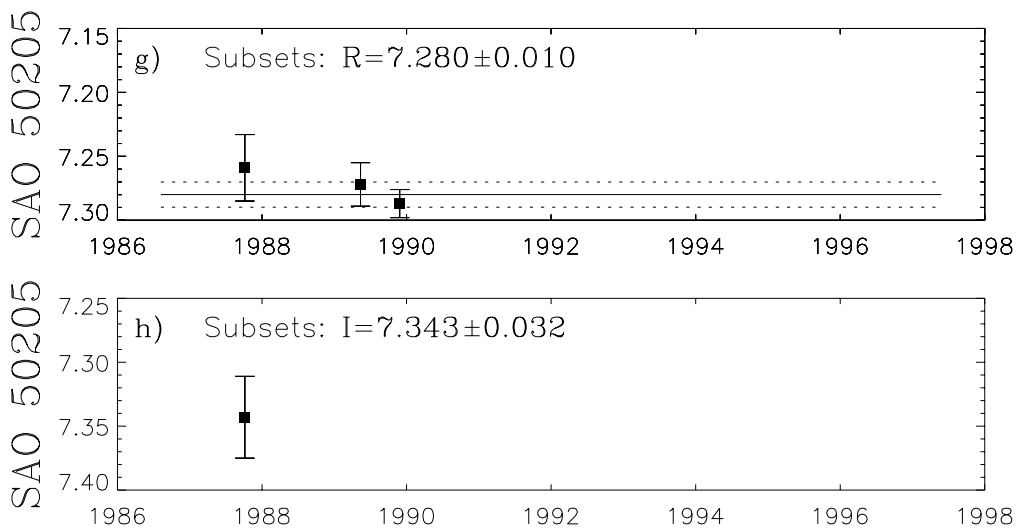

Fig. 2. The $U B V R I$ magnitudes of $\mathrm{SAO} 50205$, otherwise as in Fig. 1

57 Cyg is the only early-type $C_{2}$ in Table 1 , all others being late-type stars with convective outer envelopes possibly sustaining starspots.

\subsection{Secondary comparison star SAO 50257}

SAO 50257 (HD 199512, $\Delta_{\text {sep }}=1.7$ ) is a K1-2 giant or subgiant (Yoss 1961; Straizys et al. 1989). There are only two $V$ measurements (Table 1 ). The single $B$ measurement is inaccurate (Turon et al. 1992), but agrees with $B \sim$ $m_{\text {pg }}=7$. 6 (Fehrenbach et al. 1987). The short-term differential magnitudes SAO 50313 minus SAO 50257 were constant (Bopp et al. 1983; Kaluzny 1984). But SAO 50257 is an unsuitable $C_{2}$, because its long-term constant brightness is uncertain, and the $U R I$ magnitudes unknown.

\subsection{Secondary comparison star SAO 50326}

The spectral-type of SAO 50326 (HD 200060, $\Delta_{\text {sep }}=1.1$ ) is K2III (McClure 1970; Schmitt 1971; Straizys et al. 1989). The few $U B V$ measurements reveal no long-term variability (Table 1). Heckert \& Stewart (1992) detected no short-term variability in the differential $U B V(R I)_{\mathrm{C}}$ magnitudes SAO 50313 minus SAO 50326. But this $C_{2}$ is unsuitable, because its $R I$ magnitudes are unknown.

\section{Primary comparison stars $\left(C_{1}\right)$}

The $C_{1}$ of each SET is given in Table 3 (Col. 4). The two most frequently used $C_{1}$ are SAO 50313 and SAO 50205. The third one has been used only once $(\mathrm{SET}=2$ : SAO 50260). 


\subsection{Primary comparison star SAO 50313}

The spectral-type estimates of SAO 50313 (HD 199956, $\Delta_{\text {sep }}=1.0$ ) range between G8III and K1III (Häggkvist \& Oja 1970; Straizys et al. 1989). The short-term constant brightness was thoroughly verified by Gonzalez B. et al. (1980: their Fig. 2), who used it as $C_{1}$ in confirming that the possible pulsations of classical Am-stars cause no detectable photometric variability. We detected no short-term variability, nor periodicity between 0.4 and $50^{\mathrm{d}}$, in our new data of SAO 50313. Our measurements indicate constant long-term $B V$ brightness (Fig. 1 and Table 2). The weighted long-term $U$ mean is inaccurate $(\sim 0$. 046$)$, but comparable to the external accuracy $(\sim 0.03)$. The $\Delta U \approx 4.5$ difference between $57 \mathrm{Cyg}$ and SAO 50313 probably induces this scatter in the APT photometry. It may also indicate low accuracy for the transformations into the standard Johnson system. The $B V$ remain constant during some apparently large $U$ changes (e.g. $\mathrm{SET}=33$ and 36 ). But $U B V$ changes should correlate, were the $U$ changes induced by starspots (e.g. Paper II: Fig. 3). Only one $R$ measurement of SAO 50313 has been made, and none in $I$. In conclusion, the long-term constant brightness was established in $B V$, but not in $U$. The onset of photometric variability in late-type stars as a function of the Rossby number has been studied, e.g., by Hall (1991). Thus the determination of $v \sin i$ of this latetype $C_{1}$ might give a rough estimate of whether brightness variations due to starspots could even be expected.

\subsection{Primary comparison star SAO 50205}

SAO 50205 (HD 199206, $\Delta_{\text {sep }}=0.7$ ) is an early-type B8II close visual binary also known as ADS 14411AB (Fehrenbach et al. 1961). Estimates of the magnitude difference and the angular separation between the A and $\mathrm{B}$ components range from $\Delta V=1.70$ to 1.82 , and from 2'.53 to 2.! 80 (Rakos et al. 1982; Turon et al. 1992). This angular separation should not cause observational errors in photometry. For example, the diaphragm diameters of the APT Phoenix 10 inch telescope at the Mount Hopkins Observatory and the AZT-14 telescope at the Mount Maidanak Observatory are $60^{\prime \prime}$ and $22^{\prime \prime}$, respectively. Our new SAO 50205 data confirm constant longterm $U B V$ brightness with a high precision (Fig. 2). The three $R$ measurements are in overall agreement, but the single $I$ measurement is inaccurate. SAO 50205 has shown no short-term photometric variability (Rakos et al. 1982). No significant periodicity was detected between 0.4 and $50^{\mathrm{d}}$, nor irregular short-term variability in our extensive APT data. Hence the early-type SAO 50205 is a reliable $C_{1}$ for V 1794 Cyg.

\subsection{Primary comparison star SAO 50260}

SAO 50260 (HD 199547, K0-2III, $\Delta_{\text {sep }}=0.6$ ) is a long-period $\left(P_{\text {orb }}=2871^{\mathrm{d}} \pm 14^{\mathrm{d}}\right)$ late-type spectroscopic binary (Fehrenbach et al. 1961; Häggkvist \& Oja 1970; Griffin 1984). Short-term constant brightness has not been verified. The long-term $U B V$ magnitudes suggest no variability, but the $R I$ magnitudes are unknown (Table 2). SAO 50260 was used as $C_{1}$ only once $(\mathrm{SET}=2)$. Because the two most frequently used $C_{1}$ seem reliable (SAO 50313 and SAO 50205), using SAO 50260 as $C_{1}$ of $\mathrm{V} 1794$ Cyg is unnecessary.

\subsection{Long-term mean correction}

The long-term mean brightness correction for any $C_{1}$ (hereafter LTM-correction) consists of two parts. First, the $U B V R I$ magnitudes of $C_{1}$ used in deriving the $O$ magnitudes during any previous study are subtracted, and the "original" $\Delta m_{O-C_{1}}$ obtained. Then, the corrected longterm means of $C_{1}$ are added to these $\Delta m_{O-C_{1}}$. Here the corrected long-term means for any $C_{1}$ of V 1794 Cyg are the weighted means of Table 2 . The reasons for the LTMcorrection are evident. Firstly, the improved $U B V R I$ magnitudes of $C_{1}$ are used. Secondly, consistent long-term differential photometry relies on a constant $C_{1}$ brightness. Were different $U B V R I$ magnitudes of $C_{1}$ used during different subsets, the mean brightness level of $O$ would be inconsistent. Thirdly, $C_{1}$ is not measured during every SET. Thus the brightness must be assumed being equal to the long-term mean determined during other subsets. Our laborious $C_{1}$ and $C_{2}$ analysis for V 1794 Cyg could have been avoided, had the same combination been consistently used. In the future, only one thoroughly tested $C_{1}$ and $C_{2}$ combination should be used in the differential photometry of $\mathrm{V} 1794 \mathrm{Cyg}$. When the brightness of this combination is accurately determined, procedures like the LTM-correction become unnecessary.

\section{Previous V 1794 Cyg photometry}

The previously published photometry of V 1794 Cyg in our Table 4 consists of the standard Johnson $U B V R I$ photometry of Paper II and the $U B V(R I)_{\mathrm{C}}$ photometry by Heckert \& Stewart (1992). These are discussed separately in Sects. 5.1 and 5.2. The first part of the APT photometry in Dempsey et al. (1992) was already published in Paper II. The numerical values of the second part of these APT data are published for the first time in our paper (i.e. the APT photometry for B.W. Bopp in Sect. 6.1). Hence the data in Dempsey et al. (1992) are referred to "This paper" in our Table 3. Apart from Rodono \& Cutispoto (1992), our Table 4 contains all V 1794 Cyg photometry published before 1995 . 
Table 2. The $U B V R I$ magnitudes of all $C_{1}$ and their weighted long-term means. YEAR is the SET mean epoch

\begin{tabular}{|c|c|c|c|c|c|c|}
\hline \multicolumn{7}{|l|}{ SAO 50313} \\
\hline & $8.700 \pm 0.029$ & $7.730 \pm 0.018$ & $6.660 \pm 0.012$ & & & Landolt 1975 \\
\hline & & & $6.640 \pm 0.010$ & & & Straizys et al. 1989 \\
\hline & & $7.730 \pm 0.034$ & $6.660 \pm 0.031$ & & & Turon et al. 1992 \\
\hline 1982.84 & $8.642 \pm 0.018$ & $7.697 \pm 0.014$ & $6.624 \pm 0.016$ & & & $\mathrm{SET}=7$, Paper II \\
\hline 1983.34 & $8.695 \pm 0.042$ & $7.744 \pm 0.024$ & $6.670 \pm 0.021$ & & & $\mathrm{SET}=8$, Paper II \\
\hline 1983.64 & $8.693 \pm 0.009$ & $7.707 \pm 0.017$ & $6.643 \pm 0.014$ & & & $\mathrm{SET}=9$, Paper II \\
\hline 1984.33 & $8.674 \pm 0.074$ & $7.710 \pm 0.041$ & $6.643 \pm 0.032$ & & & $\mathrm{SET}=11$, Paper II \\
\hline 1984.70 & $8.712 \pm 0.032$ & $7.713 \pm 0.009$ & $6.634 \pm 0.005$ & & & $\mathrm{SET}=13$, Paper II \\
\hline 1985.39 & $8.687 \pm 0.046$ & $7.724 \pm 0.023$ & $6.643 \pm 0.021$ & & & $\mathrm{SET}=14$, Paper II \\
\hline 1988.74 & $8.561 \pm 0.044$ & $7.748 \pm 0.022$ & $6.667 \pm 0.031$ & & & $\mathrm{SET}=33$, Paper II \\
\hline 1988.80 & $8.621 \pm 0.039$ & $7.724 \pm 0.013$ & $6.633 \pm 0.020$ & & & $\mathrm{SET}=34$, Paper II \\
\hline 1988.86 & $8.605 \pm 0.041$ & $7.731 \pm 0.016$ & $6.642 \pm 0.020$ & & & $\mathrm{SET}=35$. Paper II \\
\hline 1988.93 & $8.572 \pm 0.041$ & $7.724 \pm 0.018$ & $6.639 \pm 0.019$ & & & $\mathrm{SET}=36$, Paper II \\
\hline 1989.36 & $8.637 \pm 0.037$ & $7.714 \pm 0.015$ & $6.626 \pm 0.020$ & & & $\mathrm{SET}=37$, Paper II \\
\hline 1989.45 & $8.629 \pm 0.048$ & $7.715 \pm 0.014$ & $6.624 \pm 0.021$ & & & $\mathrm{SET}=39$, Paper II \\
\hline 1989.72 & $8.578 \pm 0.035$ & $7.702 \pm 0.017$ & $6.632 \pm 0.020$ & & & $\mathrm{SET}=43$, This paper \\
\hline 1989.79 & $8.570 \pm 0.034$ & $7.695 \pm 0.014$ & $6.625 \pm 0.023$ & & & $\mathrm{SET}=44$. This paper \\
\hline 1989.86 & $8.596 \pm 0.037$ & $7.695 \pm 0.014$ & $6.622 \pm 0.018$ & & & $\mathrm{SET}=46$, This paper \\
\hline 1989.91 & $8.575 \pm 0.026$ & $7.681 \pm 0.017$ & $6.621 \pm 0.015$ & $5.816 \pm 0.010$ & & $\mathrm{SET}=47$, This paper \\
\hline 1989.94 & $8.642 \pm 0.036$ & $7.712 \pm 0.014$ & $6.637 \pm 0.020$ & & & $\mathrm{SET}=48$, This paper \\
\hline 1990.38 & $8.596 \pm 0.036$ & $7.703 \pm 0.012$ & $6.631 \pm 0.020$ & & & $\mathrm{SET}=49$. This paper \\
\hline 1990.45 & $8.602+0.036$ & $7.696 \pm 0.012$ & $6.626+0.020$ & & & $\mathrm{SET}=52$. This paper \\
\hline 1990.74 & $8.697 \pm 0.039$ & $7.714 \pm 0.015$ & $6.630 \pm 0.020$ & & & $\mathrm{SET}=57$, This paper \\
\hline 1990.82 & $8.704 \pm 0.036$ & $7.716 \pm 0.012$ & $6.634 \pm 0.018$ & & & $\mathrm{SET}=62$. This paper \\
\hline 1990.92 & $8.652 \pm 0.037$ & $7.719 \pm 0.015$ & $6.648 \pm 0.019$ & & & $\mathrm{SET}=65$, This paper \\
\hline 1991.38 & $8.695 \pm 0.036$ & $7.736 \pm 0.015$ & $6.639 \pm 0.019$ & & & $\mathrm{SET}=67$. This paper \\
\hline \multirow{2}{*}{$\begin{array}{l}1991.38 \\
1991.46\end{array}$} & $8.667 \pm 0.034$ & $7.721 \pm 0.011$ & $6.625 \pm 0.018$ & & & $\mathrm{SET}=70$, This paper \\
\hline & $8.659 \pm 0.046$ & $7.713 \pm 0.014$ & $6.636 \pm 0.011$ & $5.816 \pm 0.010$ & & Weighted mean for SAO 50313 \\
\hline \multicolumn{7}{|l|}{ SAO 50205} \\
\hline & & $7.330 \pm 0.035$ & $7.350 \pm 0.022$ & & & Ljunggren \& Oja 1964 \\
\hline & $7.070 \pm 0.031$ & $7.290 \pm 0.026$ & $7.330 \pm 0.022$ & & & Deutschman et al. 1976 \\
\hline & & & $7.320 \pm 0.010$ & & & Straizys et al. 1989 \\
\hline & & $7.307 \pm 0.012$ & $7.340 \pm 0.010$ & & & Turon et al. 1992 \\
\hline 1987.76 & $7.004 \pm 0.039$ & $7.313 \pm 0.035$ & $7.330 \pm 0.031$ & $7.259 \pm 0.026$ & $7.343 \pm 0.032$ & $\mathrm{SET}=28$, Paper II \\
\hline 1989.36 & & & $7.334 \pm 0.016$ & $7.272 \pm 0.017$ & & $\mathrm{SET}=38$, Paper II \\
\hline 1989.91 & $7.065 \pm 0.006$ & $7.283 \pm 0.012$ & $7.351 \pm 0.009$ & $7.287 \pm 0.011$ & & $\mathrm{SET}=47$, This paper \\
\hline 1990.40 & $7.082 \pm 0.034$ & $7.339 \pm 0.009$ & $7.348 \pm 0.021$ & & & $\mathrm{SET}=50$. This paper \\
\hline 1990.46 & $7.080 \pm 0.037$ & $7.340 \pm 0.015$ & $7.343 \pm 0.019$ & & & $\mathrm{SET}=53$, This paper \\
\hline 1990.75 & $7.088 \pm 0.033$ & $7.344 \pm 0.013$ & $7.348 \pm 0.019$ & & & $\mathrm{SET}=58$, This paper \\
\hline 1990.81 & $7.083 \pm 0.032$ & $7.339 \pm 0.012$ & $7.348 \pm 0.019$ & & & $\mathrm{SET}=61$, This paper \\
\hline 1990.92 & $7.086 \pm 0.035$ & $7.341 \pm 0.015$ & $7.345 \pm 0.021$ & & & $\mathrm{SET}=64$, This paper \\
\hline 1991.41 & $7.090+0.034$ & $7.349+0.013$ & $7.349+0.020$ & & & $\mathrm{SET}=69$. This paper \\
\hline 1991.48 & $7.083 \pm 0.032$ & $7.349 \pm 0.012$ & $7.349 \pm 0.019$ & & & $\mathrm{SET}=71$, This paper \\
\hline 1991.77 & & $7.345 \pm 0.015$ & $7.351 \pm 0.020$ & & & $\mathrm{SET}=76$. This paper \\
\hline 1991.84 & & $7.349 \pm 0.012$ & $7.352 \pm 0.020$ & & & $\mathrm{SET}=79$, This paper \\
\hline 1992.38 & & $7.350 \pm 0.013$ & $7.352 \pm 0.020$ & & & $\mathrm{SET}=80$, This paper \\
\hline 1992.48 & & $7.347+0.013$ & $7.354+0.020$ & & & $\mathrm{SET}=82$. This paper \\
\hline 1992.78 & $7.069 \pm 0.033$ & $7.346 \pm 0.015$ & $7.350 \pm 0.020$ & & & $\mathrm{SET}=88$, This paper \\
\hline 1992.89 & $7.071 \pm 0.034$ & $7.346 \pm 0.018$ & $7.351 \pm 0.018$ & & & $\mathrm{SET}=90$, This paper \\
\hline 1993.45 & $7.070 \pm 0.032$ & $7.347 \pm 0.013$ & $7.351 \pm 0.018$ & & & $\mathrm{SET}=92$, This paper \\
\hline 1993.56 & $7.068 \pm 0.033$ & $7.345 \pm 0.013$ & $7.353 \pm 0.019$ & & & $\mathrm{SET}=95$, This paper \\
\hline 1993.72 & $7.064 \pm 0.034$ & $7.341 \pm 0.014$ & $7.345 \pm 0.019$ & & & $\mathrm{SET}=100$, This paper \\
\hline 1993.81 & $7.074 \pm 0.034$ & $7.334 \pm 0.017$ & $7.353 \pm 0.023$ & & & $\mathrm{SET}=104$, This paper \\
\hline 1993.91 & $7.067 \pm 0.032$ & $7.326 \pm 0.017$ & $7.340 \pm 0.022$ & & & $\mathrm{SET}=105$, This paper \\
\hline 1994.48 & $7.072 \pm 0.032$ & $7.342 \pm 0.011$ & $7.347 \pm 0.019$ & & & $\mathrm{SET}=106$, This paper \\
\hline 1995.49 & $7.057 \pm 0.032$ & $7.349 \pm 0.012$ & $7.353 \pm 0.018$ & & & $\mathrm{SET}=111$ This paper \\
\hline 1995.76 & $7.070 \pm 0.033$ & $7.338 \pm 0.012$ & $7.345 \pm 0.018$ & & & $\mathrm{SET}=112$, This paper \\
\hline 1995.84 & $7.072 \pm 0.033$ & $7.340 \pm 0.015$ & $7.349 \pm 0.020$ & & & $\mathrm{SET}=113$, This paper \\
\hline \multirow[t]{2}{*}{1995.90} & $7.073 \pm 0.032$ & $7.343 \pm 0.015$ & $7.356 \pm 0.020$ & & & $\mathrm{SET}=114$, This paper \\
\hline & $7.068 \pm 0.010$ & $7.338 \pm 0.016$ & $7.345 \pm 0.010$ & $7.280 \pm 0.010$ & $7.343 \pm 0.032$ & Weighted mean for SAO 50205 \\
\hline \multicolumn{7}{|l|}{ SAO 50260 } \\
\hline & $9.280 \pm 0.040$ & $8.200 \pm 0.040$ & $7.070 \pm 0.040$ & & & Landolt 1975 \\
\hline & & & $7.040 \pm 0.010$ & & & Straizys et al. 1989 \\
\hline & $9.230 \pm 0.019$ & $8.190 \pm 0.016$ & $7.040 \pm 0.013$ & & & Oja 1991 \\
\hline & & $8.200 \pm 0.034$ & $7.070 \pm 0.031$ & & & Turon et al. 1992 \\
\hline & $9.239 \pm 0.019$ & $8.193 \pm 0.004$ & $7.043 \pm 0.009$ & & & Weighted mean for SAO 50260 \\
\hline
\end{tabular}




\subsection{UBVRI photometry in Paper II}

Table 3 gives the original reference of each SET in Paper II (Bopp 1982; Bopp et al. 1983; Kaluzny 1984; Nations \& Seeds 1986; Huovelin et al. 1987; Paper II). Our LTM-correction for the three $C_{1}$ in Paper II was: First, the "original" $\Delta m_{O-C_{1}}$ within each SET were obtained from the magnitudes of V $1794 \mathrm{Cyg}$ (Paper II: Table 2) by subtracting the earlier $C_{1}$ magnitudes (Paper II: Table 1). Then, the weighted $U B V R I$ means of each $C_{1}$ from Table 2 of this paper were added to these "original" $\Delta m_{O-C_{1}}$. For example, the following LTM-correction was applied to the first $V_{\text {old }, O}=7.225$ measurement of $\mathrm{V} 1794 \mathrm{Cyg}$ on HJD $=2442719.5779$. The $V$ magnitude for $\mathrm{SAO} 50313$ was $V_{\text {old, } C_{1}}=6.653$ in Paper II. The weighted mean in our Table 2 is $V_{\text {new }, C_{1}}=6.636$. Hence the first line in our Table 4 has

$V_{\text {new }, O}=V_{\text {old }, O}-V_{\text {old }, C_{1}}+V_{\text {new }, C_{1}}=7.208$.

This particular LTM-correction is small (0.017). But the LTM-corrections are larger for subsets with SAO 50205 as $C_{1}$ : 0 0. 05, 0. ${ }^{\mathrm{m}} 04,00^{\mathrm{m}} 04,0{ }^{\mathrm{m}} 02$ and 0.03 in $U B V R I$, respectively. This is partly due to the $B V R I$ magnitudes of 16 Cyg B (Moffett \& Barnes 1979) that were used in Paper II to determine the brightness of SAO 50205 during $\mathrm{SET}=28$. Another reason for this large LTM-correction was the inaccurate differential $U B$ magnitudes of SAO 50205 minus SAO 50313 during $\mathrm{SET}=38$ of Paper II, which have been excluded from our Table 2. On the other hand, the LTM-corrections in $U B V R$ for subsets with SAO 50313 as $C_{1}$ were between $0 .{ }^{\mathrm{m}} 01$ and 0.02 . Finally, SET $=2\left(C_{1}\right.$ : SAO 50260) required a LTM-correction of only 0.01 in $B V$. Some minor corrections were made to the photometry of Paper II. Three possibly erroneous observations were excluded: the two $V$ magnitudes on HJD $=2446308.8667$ and 2447320.8750, and the $B$ magnitude on HJD = 2447439.7549. In addition to these, flares were identified in four subsets of Paper II (Col. 8 of Table 4), but these will be discussed in Paper III (Sect. 3.1).

\section{2. $U B V(R I)_{\mathrm{C}}$ photometry in Heckert \& Stewart (1992)}

Heckert \& Stewart (1992) obtained differential $U B V(R I)_{\mathrm{C}}$ photometry of $\mathrm{V} 1794 \mathrm{Cyg}$ during 1990 and 1991 (SET=51 and 68). Their $C_{1}$ and $C_{2}$ were SAO 50313 and SAO 50326, respectively. The standard Johnson $U B V$ magnitudes of $\mathrm{V} 1794$ Cyg were obtained by adding the SAO 50313 values from our Table 2 to the differential magnitudes. The Cousins $R$ magnitudes were transformed into the standard Johnson system (Bessell 1979). The Cousins $I$ could not be transformed, because the $I$ magnitude of $C_{1}$ (SAO 50313) is unknown.

\section{New V 1794 Cyg photometry}

The new photometry of V 1794 Cyg was made between July, 1989 and December, 1995 at the Mount Hopkins, the Mount Maidanak, the Mount Laguna, the Kvistaberg, and the Royal Swedish La Palma Observatories (Table 3: "This paper").

\subsection{Mount Hopkins Observatory (APT)}

The first part of new data is the differential $U B V$ photometry made at the Mount Hopkins Observatory between September, 1989 and December, 1995 with the (Automatic Photoelectric Telescope) Phoenix 10 inch telescope. Each observation is a mean of three ten second integrations. The average internal standard deviation of the differential magnitudes was $0{ }^{\mathrm{m}} 008,0{ }^{\mathrm{m}} 004$ and $0{ }^{\mathrm{m}} 005$ in $U, B$ and $V$, respectively. All observations with an internal error greater than $0{ }^{\mathrm{m}} 02$ are automatically discarded by the APT. The respective external errors in $U B V$ are 0.023 , 0.014 and 0.012 (e.g. Strassmeier \& Hall 1988b; Boyd et al. 1990). These external errors agree with the accuracy for the $C_{1}$ magnitudes during individual subsets of APT data (see Table 2). The APT suffered from problems with the $U$-band filter between July, 1991 and September, 1992. Thus only the $B V$-photometry of that time interval is given in Table 4 . The APT observing routines have been described in detail, e.g., by Boyd et al. (1984). The $C_{1}$ for L. Jetsu was SAO 50205, while that for B.W. Bopp and H.L. Nations was SAO 50313. The long-term $U B V$ magnitudes of these two $C_{1}$ (Table 2) were added to the differential magnitudes. The $C_{2}$ in all new APT photometry was 57 Cyg. Every SET of these new APT data has the same $C_{1}$, i.e. overlapping data with different $C_{1}$ were not combined (e.g. SET=64 and 65). This ensures the data homogeneity, and if necessary, enables simple future LTMcorrections.

\subsection{Mount Maidanak Observatory}

The second part of new data is absolute $U B V R$ photometry made at the Mount Maidanak Observatory between July, 1989 and September, 1994. The two most frequently used $C_{1}$, SAO 50313 and SAO 50205, were also observed in November, 1989 (Table 2: SET=47). The observations were made with the $48 \mathrm{~cm} \mathrm{AZT-14} \mathrm{telescope.} \mathrm{Each} \mathrm{ob-}$ servation in Table 4 is an average of $3-5$ ten second integrations in each $U B V R$ passband. A set of standard stars was observed to determine the nightly extinction coefficients and the transformation into the standard Johnson $U B V R$ system. The upper limit of external errors for the standard Johnson $B V R( \pm 0.015)$ and $U\left( \pm 0 . \mathrm{m}^{\mathrm{m}} 030\right)$ magnitudes have been determined, e.g., by Kilyachkov \& Shevchenko (1976) and Shevchenko (1980), who described 
Table 3. The collected photometry of V 1794 Cyg: The observing time interval, the subset number (SET), the observatory, the primary comparison star $\left(C_{1}\right)$, the secondary comparison star $\left(C_{2}\right)$, the reference and the number of observing nights $(N)$

\begin{tabular}{|c|c|c|c|c|c|c|}
\hline Interval & SET & Observatory & $C_{1}$ & $C_{2}$ & Reference & $N$ \\
\hline 3. -12.11 .1975 & 1 & McDonald Observatory & SAO 50313 & - & Bopp 1982 & 7 \\
\hline 5. -11.10 .1976 & 2 & Kitt Peak National Observatory & SAO 50260 & - & Bopp et al. 1983 & 6 \\
\hline 26.6. -17.8 .1980 & 3 & Cloudcroft Observatory & SAO 50313 & SAO 50257 & Bopp et al. 1983 & 14 \\
\hline 12.9. -13.10 .1980 & 4 & Cloudcroft Observatory & SAO 50313 & SAO 50257 & Bopp et al. 1983 & 9 \\
\hline 11.5.-18.6.1981 & 5 & Cloudcroft Observatory & SAO 50313 & SAO 50257 & Bopp et al. 1983 & 4 \\
\hline 15.9.-8.10.1982 & 6 & Crimean Astrophysical Observatory & SAO 50205 & - & Huovelin et al. 1987 & 10 \\
\hline 2.- -5.11 .1982 & 7 & Kitt Peak National Observatory & SAO 50313 & - & Paper II & 4 \\
\hline 23.4.-31.5.1983 & 8 & Kitt Peak National Observatory & SAO 50313 & - & Paper II & 6 \\
\hline 22.- -24.8 .1983 & 9 & Kitt Peak National Observatory & SAO 50313 & - & Paper II & 3 \\
\hline 8. -16.9 .1983 & 10 & Crimean Astrophysical Observatory & SAO 50205 & - & Huovelin et al. 1987 & 8 \\
\hline 20.3.-17.5.1984 & 11 & Kitt Peak National Observatory & SAO 50313 & - & Paper II & 2 \\
\hline 17. -29.8 .1984 & 12 & Ostrowik Observatory & SAO 50313 & SAO 50257 & Kaluzny 1984 & 9 \\
\hline 5.9.-10.11.1984 & 13 & Kitt Peak National Observatory & SAO 50313 & - & Paper II & 4 \\
\hline 30.4.-9.6.1985 & 14 & Kitt Peak National Observatory & SAO 50313 & - & Paper II & 8 \\
\hline 6.8.-4.9.1985 & 15 & Mount Hopkins Observatory & SAO 50313 & - & Nations \& Seeds 1986 & 16 \\
\hline 5.9.-4.10.1985 & 16 & Mount Hopkins Observatory & SAO 50313 & - & Nations \& Seeds 1986 & 20 \\
\hline $9.10 .-1.11 .1985$ & 17 & Mount Hopkins Observatory & SAO 50313 & - & Nations \& Seeds 1986 & 17 \\
\hline 2.-22.11.1985 & 18 & Mount Hopkins Observatory & SAO 50313 & - & Nations \& Seeds 1986 & 13 \\
\hline 1. -27.12 .1985 & 19 & Mount Hopkins Observatory & SAO 50313 & - & Nations \& Seeds 1986 & 13 \\
\hline 3. -29.5 .1986 & 20 & Mount Hopkins Observatory & SAO 50313 & $57 \mathrm{Cyg}$ & Paper II & 12 \\
\hline 5. -20.6 .1986 & 21 & Mount Hopkins Observatory & SAO 50313 & $57 \mathrm{Cyg}$ & Paper II & 13 \\
\hline 5. -17.9 .1986 & 22 & Crimean Astrophysical Observatory & SAO 50205 & - & Paper II & 7 \\
\hline 28.9.-28.11.1986 & 23 & Mount Hopkins Observatory & SAO 50313 & $57 \mathrm{Cyg}$ & Paper II & 16 \\
\hline 3. -30.5 .1987 & 24 & Mount Hopkins Observatory & SAO 50313 & $57 \mathrm{Cyg}$ & Paper II & 9 \\
\hline 16.6. -8.7 .1987 & 25 & Mount Hopkins Observatory & SAO 50313 & 57 Cyg & Paper II & 10 \\
\hline 11. -19.8 .1987 & 26 & Crimean Astrophysical Observatory & SAO 50205 & - & Paper II & 5 \\
\hline 28.9.-27.10.1987 & 27 & Mount Hopkins Observatory & SAO 50313 & 57 Cyg & Paper II & 13 \\
\hline 4. -12.10 .1987 & 28 & Crimean Astrophysical Observatory & SAO 50205 & 16 Cyg B & Paper II & 5 \\
\hline 8.11.-23.12.1987 & 29 & Mount Hopkins Observatory & SAO 50313 & 57 Cyg & Paper II & 10 \\
\hline $25.4 .-22.5 .1988$ & 30 & Mount Hopkins Observatory & SAO 50313 & $57 \mathrm{Cyg}$ & Paper II & 16 \\
\hline 28.5.-17.6.1988 & 31 & Mount Hopkins Observatory & SAO 50313 & 57 Cyg & Paper II & 13 \\
\hline 5.9. -17.10 .1988 & 32 & Crimean Astrophysical Observatory & SAO 50205 & - & Paper II & 8 \\
\hline 11.9.-6.10.1988 & 33 & Mount Hopkins Observatory & SAO 50313 & 57 Cyg & Paper II & 20 \\
\hline 7.10.-1.11.1988 & 34 & Mount Hopkins Observatory & SAO 50313 & 57 Cyg & Paper II & 14 \\
\hline 2. -21.11 .1988 & 35 & Mount Hopkins Observatory & SAO 50313 & 57 Cyg & Paper II & 17 \\
\hline 28.11.-25.12.1988 & 36 & Mount Hopkins Observatory & SAO 50313 & $57 \mathrm{Cyg}$ & Paper II & 18 \\
\hline 28.4.-27.5.1989 & 37 & Mount Hopkins Observatory & SAO 50313 & 57 Cyg & Paper II & 15 \\
\hline 11.-15.5.1989 & 38 & Crimean Astrophysical Observatory & SAO 50205 & SAO 50313 & Paper II & 3 \\
\hline 31.5.-24.6.1989 & 39 & Mount Hopkins Observatory & SAO 50313 & $57 \mathrm{Cyg}$ & Paper II & 14 \\
\hline 3.7.-1.8.1989 & 40 & Mount Maidanak Observatory & Absolute & Absolute & This paper & 27 \\
\hline 2.-31.8.1989 & 41 & Mount Maidanak Observatory & Absolute & Absolute & This paper & 28 \\
\hline 1. -30.9 .1989 & 42 & Mount Maidanak Observatory & Absolute & Absolute & This paper & 22 \\
\hline 10. -30.9 .1989 & 43 & Mount Hopkins Observatory & SAO 50313 & $57 \mathrm{Cyg}$ & This paper & 11 \\
\hline 1. -28.10 .1989 & 44 & Mount Hopkins Observatory & SAO 50313 & $57 \mathrm{Cyg}$ & This paper & 12 \\
\hline 3. -28.10 .1989 & 45 & Mount Maidanak Observatory & Absolute & Absolute & This paper & 13 \\
\hline 1. -20.11 .1989 & 46 & Mount Hopkins Observatory & SAO 50313 & $57 \mathrm{Cyg}$ & This paper & 15 \\
\hline 2. -30.11 .1989 & 47 & Mount Maidanak Observatory & Absolute & Absolute & This paper & 13 \\
\hline 23.11.-22.12.1989 & 48 & Mount Hopkins Observatory & SAO 50313 & 57 Cyg & This paper & 11 \\
\hline 4. -31.5 .1990 & 49 & Mount Hopkins Observatory & SAO 50313 & 57 Cyg & This paper & 11 \\
\hline 4.5.-2.6.1990 & 50 & Mount Hopkins Observatory & SAO 50205 & 57 Cyg & This paper & 11 \\
\hline 16. -26.5 .1990 & 51 & Mount Laguna Observatory & SAO 50313 & SAO 50326 & Heckert \& Stewart 1992 & 6 \\
\hline 1. -26.6 .1990 & 52 & Mount Hopkins Observatory & SAO 50313 & 57 Cyg & This paper & 11 \\
\hline 5. -26.6 .1990 & 53 & Mount Hopkins Observatory & SAO 50205 & 57 Cyg & This paper & 11 \\
\hline 6.6.-2.7.1990 & 54 & Mount Maidanak Observatory & Absolute & Absolute & This paper & 20 \\
\hline 8.7.-8.8.1990 & 55 & Mount Maidanak Observatory & Absolute & Absolute & This paper & 27 \\
\hline 9.8.-9.9.1990 & 56 & Mount Maidanak Observatory & Absolute & Absolute & This paper & 26 \\
\hline 9.9.-12.10.1990 & 57 & Mount Hopkins Observatory & SAO 50313 & 57 Cyg & This paper & 9 \\
\hline 10.9.-11.10.1990 & 58 & Mount Hopkins Observatory & SAO 50205 & $57 \mathrm{Cyg}$ & This paper & 9 \\
\hline 10. -28.9 .1990 & 59 & Mount Maidanak Observatory & Absolute & Absolute & This paper & 19 \\
\hline 4.10.-7.11.1990 & 60 & Mount Maidanak Observatory & Absolute & Absolute & This paper & 15 \\
\hline
\end{tabular}


Table 3. continued

\begin{tabular}{|c|c|c|c|c|c|c|}
\hline Interval & SET & Observatory & $C_{1}$ & $C_{2}$ & Reference & $N$ \\
\hline 12.10.-9.11.1990 & 61 & Mount Hopkins Observatory & SAO 50205 & 57 Cyg & This paper & 17 \\
\hline 13.10.-15.11.1990 & 62 & Mount Hopkins Observatory & SAO 50313 & 57 Cyg & This paper & 24 \\
\hline 8.11.-8.12.1990 & 63 & Mount Maidanak Observatory & Absolute & Absolute & This paper & 17 \\
\hline 12.11.-19.12.1990 & 64 & Mount Hopkins Observatory & SAO 50205 & 57 Cyg & This paper & 17 \\
\hline 16.11.-20.12.1990 & 65 & Mount Hopkins Observatory & SAO 50313 & 57 Cyg & This paper & 14 \\
\hline 15.12.1990-14.1.1991 & 66 & Mount Maidanak Observatory & Absolute & Absolute & This paper & 11 \\
\hline 30.4.-30.5.1991 & 67 & Mount Hopkins Observatory & SAO 50313 & $57 \mathrm{Cyg}$ & This paper & 15 \\
\hline 12.-26.5.1991 & 68 & Mount Laguna Observatory & SAO 50313 & SAO 50326 & Heckert \& Stewart 1992 & 11 \\
\hline 15.5.-8.6.1991 & 69 & Mount Hopkins Observatory & SAO 50205 & 57 Cyg & This paper & 17 \\
\hline 3.-30.6.1991 & 70 & Mount Hopkins Observatory & SAO 50313 & 57 Cyg & This paper & 20 \\
\hline 11.6.-3.7.1991 & 71 & Mount Hopkins Observatory & SAO 50205 & 57 Cyg & This paper & 14 \\
\hline 11.6. -12.7 .1991 & 72 & Mount Maidanak Observatory & Absolute & Absolute & This paper & 19 \\
\hline 15.7.-15.8.1991 & 73 & Mount Maidanak Observatory & Absolute & Absolute & This paper & 26 \\
\hline 16.8.-18.9.1991 & 74 & Mount Maidanak Observatory & Absolute & Absolute & This paper & 27 \\
\hline 19.9.-18.10.1991 & 75 & Mount Maidanak Observatory & Absolute & Absolute & This paper & 13 \\
\hline 29.9.-20.10.1991 & 76 & Mount Hopkins Observatory & SAO 50205 & 57 Cyg & This paper & 18 \\
\hline 11.10.1991 & 77 & Royal Swedish La Palma Observatory & Absolute & Absolute & This paper & 1 \\
\hline 21.10.-25.11.1991 & 78 & Mount Maidanak Observatory & Absolute & Absolute & This paper & 13 \\
\hline 26.10.-17.11.1991 & 79 & Mount Hopkins Observatory & SAO 50205 & 57 Cyg & This paper & 15 \\
\hline 6.5.-6.6.1992 & 80 & Mount Hopkins Observatory & SAO 50205 & 57 Cyg & This paper & 13 \\
\hline 12.-20.5.1992 & 81 & Mount Laguna Observatory & SAO 50313 & SAO 50326 & This paper & 6 \\
\hline 9.6.-6.7.1992 & 82 & Mount Hopkins Observatory & SAO 50205 & $57 \mathrm{Cyg}$ & This paper & 21 \\
\hline 15.7.-14.8.1992 & 83 & Mount Maidanak Observatory & Absolute & Absolute & This paper & 28 \\
\hline 6.-9.8.1992 & 84 & Mount Laguna Observatory & SAO 50313 & SAO 50326 & This paper & 2 \\
\hline 17.8.-18.9.1992 & 85 & Mount Maidanak Observatory & Absolute & Absolute & This paper & 23 \\
\hline 6. -10.9 .1992 & 86 & Royal Swedish La Palma Observatory & Absolute & Absolute & This paper & 4 \\
\hline 19.9.-19.10.1992 & 87 & Mount Maidanak Observatory & Absolute & Absolute & This paper & 18 \\
\hline 1.- -23.10 .1992 & 88 & Mount Hopkins Observatory & SAO 50205 & 57 Cyg & This paper & 17 \\
\hline 22.10.-11.11.1992 & 89 & Mount Maidanak Observatory & Absolute & Absolute & This paper & 11 \\
\hline 2.11.-1.12.1992 & 90 & Mount Hopkins Observatory & SAO 50205 & $57 \mathrm{Cyg}$ & This paper & 11 \\
\hline 26.11.-29.12.1992 & 91 & Mount Maidanak Observatory & Absolute & Absolute & This paper & 4 \\
\hline 24.5.-30.6.1993 & 92 & Mount Hopkins Observatory & SAO 50205 & $57 \mathrm{Cyg}$ & This paper & 26 \\
\hline 2. -29.6 .1993 & 93 & Mount Maidanak Observatory & Absolute & Absolute & This paper & 14 \\
\hline 6.-31.7.1993 & 94 & Mount Maidanak Observatory & Absolute & Absolute & This paper & 21 \\
\hline 24. -27.7 .1993 & 95 & Mount Hopkins Observatory & SAO 50205 & 57 Cyg & This paper & 4 \\
\hline 26.7.-2.8.1993 & 96 & Mount Laguna Observatory & SAO 50313 & SAO 50326 & This paper & 6 \\
\hline 1. -28.8 .1993 & 97 & Mount Maidanak Observatory & Absolute & Absolute & This paper & 18 \\
\hline 5. -25.8 .1993 & 98 & Kvistaberg Observatory & Absolute & Absolute & This paper & 3 \\
\hline 30.8.-24.9.1993 & 99 & Mount Maidanak Observatory & Absolute & Absolute & This paper & 21 \\
\hline 3.9. -3.10 .1993 & 100 & Mount Hopkins Observatory & SAO 50205 & 57 Cyg & This paper & 19 \\
\hline 9. -10.9 .1993 & 101 & Royal Swedish La Palma Observatory & Absolute & Absolute & This paper & 1 \\
\hline 18.9.-27.10.1993 & 102 & Kvistaberg Observatory & Absolute & Absolute & This paper & 6 \\
\hline 28.9.-28.10.1993 & 103 & Mount Maidanak Observatory & Absolute & Absolute & This paper & 17 \\
\hline 13.10.-1.11.1993 & 104 & Mount Hopkins Observatory & SAO 50205 & 57 Cyg & This paper & 9 \\
\hline 21.11.-15.12.1993 & 105 & Mount Hopkins Observatory & SAO 50205 & $57 \mathrm{Cyg}$ & This paper & 9 \\
\hline 8.6. -15.7 .1994 & 106 & Mount Hopkins Observatory & SAO 50205 & 57 Cyg & This paper & 15 \\
\hline 13.6.-8.7.1994 & 107 & Mount Maidanak Observatory & Absolute & Absolute & This paper & 20 \\
\hline 11.7.-5.8.1994 & 108 & Mount Maidanak Observatory & Absolute & Absolute & This paper & 21 \\
\hline 7.8.-1.9.1994 & 109 & Mount Maidanak Observatory & Absolute & Absolute & This paper & 17 \\
\hline 2.-28.9.1994 & 110 & Mount Maidanak Observatory & Absolute & Absolute & This paper & 14 \\
\hline 9.6.-11.7.1995 & 111 & Mount Hopkins Observatory & SAO 50205 & 57 Cyg & This paper & 21 \\
\hline 21.9.-19.10.1995 & 112 & Mount Hopkins Observatory & SAO 50205 & 57 Cyg & This paper & 22 \\
\hline 20.10.-16.11.1995 & 113 & Mount Hopkins Observatory & SAO 50205 & $57 \mathrm{Cyg}$ & This paper & 16 \\
\hline 17.11.-15.12.1995 & 114 & Mount Hopkins Observatory & SAO 50205 & $57 \mathrm{Cyg}$ & This paper & 16 \\
\hline
\end{tabular}


the Mount Maidanak Observatory photometric observing routines, instrumentation, reductions, and transformations.

\subsection{Mount Laguna Observatory}

The third part of new data consists of the differential $U B V(R I)_{\mathrm{C}}$ photometry made between May, 1992 and August, 1993 with the 24 inch telescope at the Mount Laguna Observatory. The comparison stars SAO $50313\left(C_{1}\right)$ and SAO $50326\left(C_{2}\right)$ were as in Heckert \& Stewart (1992). Each observation is an average of four 10 seconds integrations through a diaphragm of $19^{\prime \prime}$ or $26^{\prime \prime}$, depending on the seeing conditions and/or background brightness. The internal error is $\leq 0 \mathrm{~m} 01$ in every passband. The standard stars (Landolt 1983) were observed to determine the transformation into the standard Johnson $U B V$ and Cousins $R I$ systems. The weighted mean $U B V R$ magnitudes of SAO 50313 in Table 2 were used to derive the magnitudes of V 1794 Cyg. The Cousins $R$ magnitudes were transformed into the standard Johnson system (Bessel 1979). The standard Johnson $I$ magnitudes could not be derived, because $I$ of SAO 50313 is unknown. The Mount Laguna Observatory observing routines and instrumentation have been described, e.g., in Heckert \& Stewart (1992) and Heckert (1993).

\subsection{Kvistaberg Observatory and Royal Swedish La Palma Observatory}

The fourth part of the new data is absolute $U B V$ photometry made between October, 1991 and October, 1993 with the $40 \mathrm{~cm}$ telescope at Kvistaberg Observatory and the $60 \mathrm{~cm}$ telescope at Royal Swedish La Palma Observatory. These observations were obtained during a survey of medium magnitude $U B V$ standard stars at positive declinations (between $15^{\circ}$ and $45^{\circ}$ ), i.e. V $1794 \mathrm{Cyg}$ was "accidentally" included into a list of standard stars, before being identified as a variable. As an exception, the first observation $(\mathrm{SET}=77)$ was made during a study of late-type stars in the Milky Way. The reductions by T. Oja are essentially the same for both instruments. Each observation is a mean of three ten second integrations. The transformation into the standard Johnson $U B V$ is determined, and continuously verified, with respect to standard star measurements, as described, e.g., by Oja (1983). The external accuracy is about $0^{\mathrm{m}} 021$ in $V$, and $0{ }^{\mathrm{m}} 011$ in $B-V$ and $U-B$. The $V$ magnitudes of V $1794 \mathrm{Cyg}$ were reduced relative to three standard stars using the values given in the parenthesis: $\mathrm{BD}+44^{\circ} 3604(V=9.420), \mathrm{BD}+45^{\circ} 3310$ $(V=8.647)$, and $\mathrm{BD}+54^{\circ} 3220(V=8.297)$, while the $B-V$ and $U-B$ of $\mathrm{V} 1794 \mathrm{Cyg}$ are absolute.

\section{Conclusions}

Achieving something comparable to the sunspot-record for any late-type star requires uninterrupted and coordinated photometric observing programs. The CaII H\&K emission measurements for about 100 solar-type mainsequence stars already extend over a quarter of a century (e.g. Wilson 1978; Saar \& Baliunas 1992), and more than a decade of photometry of these stars has been made (e.g. Radick et al. 1990; Radick 1992). The necessity for long-term photometric programs for an extensive sample of all types of chromospherically active stars (e.g. pre- and post-main-sequence stars) has been emphasized, e.g., by Hall (1991). The automatic photoelectric telescopes could perform such a coordinated effort, but only smaller samples have been studied (e.g. Strassmeier \& Hall 1988a,b; Henry et al. 1995). Systematic collection and easy availability of photometry obtained with automated telescopes should therefore receive utmost attention. The data time span could be extended with photographic plate archives (e.g. Hartmann et al. 1979). Very intensive photometry of V 1794 Cyg has been carried out, and our data now covers about 20 years. In observing times, the increment from Paper I is about $207 \%$. The number of individual $U B V R I$ measurements has increased even more $(\sim 286 \%)$.

Absolute photometry relies on a selected set of standard stars, but differential photometry requires constant brightness for $C_{1}$ and $C_{2}$. The best combination for differential photometry of V $1794 \mathrm{Cyg}$ is: $C_{1}=\mathrm{SAO} 50313$ or SAO 50205, and $C_{2}=57$ Cyg. No short- or long-term photometric variability was detected in these comparison stars. Not only will this combination simplify new photometry of V $1794 \mathrm{Cyg}$, but more importantly, it ensures the homogeneity of all collected photometry. The accuracy in $U$ for SAO 50313 is lower than for SAO 50205. The colour index difference between an early-type $C_{1}$ (i.e. SAO 50205) and a late-type variable does require secondary extinction corrections in differential photometry, especially in $U B$. Yet, the choice of a late-type $C_{1}$ (i.e. SAO 50313) may have unfortunate consequences. For example, Hall (1976) discusses the case, where the longperiod variable HK Lac was adopted as $C_{1}$ for the RS CVn star AR Lac.

Our collection of V 1794 Cyg photometry, as well as of all $C_{1}$ and $C_{2}$, was terminated on 1995 . Analysing 114 subsets would have otherwise been impossible, because any new $C_{1}$ or $C_{2}$ measurements would have required LTMcorrections in several subsets, i.e. revision of Table 4. For example, who could have foretold the planetary companion detection in one of our $C_{2}$, namely 16 Cyg B (Cochran et al. 1997)? For the purposes of our study, the "good news" about $16 \mathrm{Cyg} \mathrm{B}$ are that we used this $C_{2}$ only once, and that planetary transit detections in stellar photometry are difficult (Henry et al. 1997). Finally, it was impossible to determine the accuracy of every individual $U B V R I$ measurement of V 1794 Cyg in Table 4. Hence 
we conclude that reasonable mean external accuracy estimates for all collected photometry are $0 .{ }^{\mathrm{m}} 015$ in $B V R I$ and 0.030 in $U$. A prolonged time series of homogeneous standard Johnson UBVRI photometry of V 1794 Cyg has been collected and pre-processed. These data are analysed in Paper III.

Acknowledgements. The work was partially supported by the EC Human Capital and Mobility (Networks) project "Late type stars: activity, magnetism, turbulence" No. ERBCHRXCT940483. Dr. M. Seeds and the APT Service are acknowledged of high quality photometry. This research has made use of the Simbad-database operated at CDS, Strasbourg, France.

\section{References}

Argue A.N., 1966, MNRAS 133, 475

Batten A.H., Fletcher J.M., Mann P.J., 1978, Publ. Dominion Astrophys. Obs. Victoria 15, 121

Bessell M.S., 1979, PASP 91, 589

Bopp B.W., 1982, in Second Cambridge Workshop on Cool Stars, Stellar systems, and the Sun, Giampapa M.S., Golub L. (eds.). SAO Special Report 392, p. 207

Bopp B.W., Rucinski S.M., 1981, in Fundamental Problems in the Theory of Stellar Evolution, Sugimoto D., Lamb D.Q., Schramm D.N. (eds.). IAU Symp. 93, p. 177

Bopp B.W., Stencel R.E., 1981, ApJ 247, L131

Bopp B.W., Africano J.L., Stencel R.E., Noah P.V., Klimke A., 1983, ApJ 275, 691

Boyd L.J., Genet R.M., Hall D.S., 1984, IAPPP Comm. 15, 20

Boyd L.J., Genet R.M., Hall D.S., Busby R.M., Henry G.W., 1990, IAPPP Comm. 42, 44

Campins H., Rieke G.H., Lebofsky M.J., 1985, AJ 90, 896

Crawford D.L., Barnes J.V., Golson J.C., 1971, AJ 76, 1058

Cochran W.D., Hatzes A.P., Butler R.P., Marcy G.W., 1997, ApJ 483, 457

Dempsey R.C., Bopp B.W., Strassmeier K.G., et al., 1992, ApJ 392, 187

Deutschman W.A., Davis R.J., Schild R.E., 1976, ApJS 30, 97

Eggen O.J., 1968, Royal Obs. Bull. 137

Fabregat J., Reglero V., 1990, A\&AS 82, 531

Fehrenbach Ch., Petit M., Cruvellier G., Peyrin Y., 1961, Journal des Observateurs 44, 233

Fehrenbach Ch., Duflot M., Burnage R., et al., 1987, A\&AS 71, 275

Fekel F.C., 1997, PASP 109, 514

Giuricin G., Mardirossian F., Mezzetti M., 1984, ApJS 54, 421

Gonzalez B.S.F., Warman J., Pena J.H., 1980, AJ 85, 1361

Grewing M., Lequeux J., Pottasch S.R., 1992, A\&A 266, E1

Griffin R.F., 1984, Observatory 104, 6

Hall D.S., 1976, in Multiple Periodic Variable Stars, Fitch W.S. (ed.). IAU Coll. 29. Reidel, Dordrecht, p. 287

Hall D.S., 1991, in The Sun and Cool Stars: activity, magnetism, dynamos, Tuominen I, Moss D., Rüdiger G.R. (eds.). IAU Coll. 130. Springer-Verlag, Heidelberg, p. 353

Hartmann L., Londono C., Phillips M.J., 1979, ApJ 229, 183

Heckert P.A., Stewart M.C., 1992, IBVS 3717
Heckert P.A., 1993, IBVS 3954

Henry G.W., Eaton J.A., Hamer J., Hall D.S., 1995, ApJS 97, 513

Henry G.W., Baliunas S.L., Donahue R.A., Soon W.H., Saar S.H., 1997, ApJ 474, 503

Herbig G.H., 1958, ApJ 128, 259

Hilditch R.W., 1973, MNRAS 164, 101

Huenemoerder D.P., 1986, AJ 92, 673

Huovelin J., Piirola V., Vilhu O., Efimov Yu. S., Shakhovskoy N.M., 1987, A\&A 176, 83

Häggkvist L., Oja T., 1970, A\&AS 1, 199

Jetsu L., Huovelin J., Tuominen I., et al., 1990a, A\&A 236, 423 (Paper I)

Jetsu L., Huovelin J., Tuominen I., et al., 1990b, A\&AS 85, 813 (Paper II)

Jetsu L., Pelt J., Tuominen I., 1999, A\&A (in press) (Paper III)

Johnson H.L., Morgan W.W., 1953, ApJ 117, 313

Johnson H.L., Mitchell R.I., Iriarte B., Wiśniewski W.Z., 1966, LPL Comm. 63, 99

Kaluzny J., 1984, IBVS 2627

Kilyachkov N.N., Shevchenko V.S., 1976, Pis'ma Astron Zh. 2, 494 (Sov. Astron. Lett 2, 193)

Landolt A.U., 1975, PASP 87, 379

Landolt A.U., 1983, AJ 88, 439

Ljunggren B., Oja T., 1964, Ark. Astr. 3, 439

McClure R.D., 1970, AJ 75, 41

Moffett T.J., Barnes T.G., 1979, PASP 91, 180

Nations H.L., Seeds M.A., 1986, IAPPP Commun. 25, 56

Neckel H., 1986, A\&A 167, 97

Oja T., 1983, A\&AS 52, 131

Oja T., 1991, A\&AS 89, 415

Olsen E.H., 1983, A\&AS 54, 55

Percy J.R., Welch D.L., 1983, PASP 95, 491

Radick R.R., 1992, in The Solar Cycle, Harvey K.L. (ed.). ASP Conf. Ser. 27, 450

Radick R.R., Lockwood G.W., Baliunas S.L., 1990, Sci 247, 39

Rakos K.D., Albrecht R., Jenkner H., et al., 1982, A\&AS 47, 221

Rakos K.D., Franz O.G., 1988, Ap\&SS 142, 123

Rodono M., Cutispoto G., 1992, A\&AS 95, 55

Saar S.H., Baliunas S.L., 1992, in The Solar Cycle, Harvey K.L. (eds.). ASP Conf. Ser. 27, 150

Schmitt J.L., 1971, ApJ 163, 75

Shevchenko V.S., 1980, Sov. Astron. Zh. 57, 1162 (SvA 24, 670)

Skiff B.A., 1993, IBVS 3906

Stetson P.B., 1991, AJ 102, 589

Straizys V., Meistas E., Vansevicius V., Goldberg E.P., 1989, Vilnius Astron. Obs. Biul. 83, 3

Strassmeier K.G., Hall D.S., 1988a, ApJS 67, 439

Strassmeier K.G., Hall D.S., 1988b, ApJS 67, 453

Tedesco E.F., Tholen D.J., Zellner B., 1982, AJ 87, 1585

Tolbert C.R., 1964, ApJ 139, 1105

Turon C., Crézé M., Egret D., et al., 1992, The Hipparcos Input Catalogue, ESA SP-1136

Willson R.C., Hudson H.S., 1991, Nat 351, 42

Wilson O.C., 1978, ApJ 226, 379

Yoss K.M., 1961, ApJ 134, 809 